\title{
Isolation of Greater Amberjack Microsatellite DNA and Their Application as Genetic Marker to Species of Genus Seriola from Japan
}

\author{
Estu Nugroho and Nobuhiko Taniguchi* \\ Faculty of Agriculture, Tohoku University, Amamiya-cho, Aoba, Sendai, 981-8555 Japan \\ (Received August 3, 1998)
}

\begin{abstract}
Six primer sets were designed from the greater amberjack, Seriola dumerili, and tested to greater amberjack and three other species of genus Seriola. The primer sets tested have only scorable product in samples from the same genus and may not be available to amplify DNA from the other genera. Four of those loci were polymorphic for greater amberjack, and three loci were polymorphic for other species of genus Seriola, with number of alleles ranging from 2 to 16 , and observed heterozygosities between 0.350 to 0.800 . Species $S$. lalandi has the highest variability, while the lowest belongs to species $S$. rivoliana. Genetic distance, calculated over those loci for all species samples, shows a clear clarification of species according to the taxonomic arrangement. These loci show potential as genetic markers for assessment of genetic variability and population genetic study of species of genus Seriola.
\end{abstract}

Key words: microsatellite, DNA polymorphism, greater amberjack, Seriola

Carangids are classified into 23 genera and four subfamilies, ${ }^{12}$ which also include fusiform species adapted to long migration and compressiform species adapted to the life in coastal areas. There are about 50 or more species caught by commercial and sport fishing in the coastal and offshore areas of Japan. Some species of the genus Seriola ( $S$. dumerili, $S$. rivoliana, $S$. quinqueradiata and $S$. lalandi) are important in aquaculture in several prefectures of Japan. As the seed fish have been collected from the natural waters and sometimes imported from other countries, the increase of both activities may exert a growing pressure on the natural population through reducing genetic variability or changing the population structure of those species. $\mathrm{FAO}^{2)}$ recommended some measures in order to conserve the aquatic genetic resources of natural populations, one measure is pursuant to eventual determination of the genetic structure of the natural population.

One alternative to gathering that information on these species is the use of molecular genetic markers. ${ }^{3)}$ Recently, a number of easily assayable and highly variable genetic markers have been developed. From several genetic markers, microsatellite is increasingly being used in genetic studies due to the high polymorphism and is generally inherited in a Mendelian way.

As part of an effort to develop an easily assayable and highly variable genetic marker for population study of Seriola, greater amberjack, we have cloned and sequenced microsatellite loci from the greater amberjack genome. Four of those were used to assess the allelic variation within and among four species of genus Seriola from Japan areas. Assessing the baseline variation at microsatellite loci is a prerequisite for determination of genetic structure and levels of variation present of their natural population.

\section{Materials and Methods}

\section{Samples}

Four species of Seriola samples were collected from Kochi prefecture during October-December 1995 and October-December 1997, i.e. S. dumerili $(n=40)$, S. rivoliana $(n=20), S$. quinqueradiata $(n=40)$, and $S$. lalandi $(n=40)$. Two species of other genera (Decapterus maruadsi, $n=20$, and Uraspis helvola, $n=20$ ) were also used to test the capability of primers revealed from the greater amberjack genome.

\section{Cloning and Sequencing of Microsatellites}

Greater amberjack genomic DNA was extracted from blood and fin clippings using a standard SDS-phenol/chloroform method. ${ }^{4)}$ Cloning and sequencing procedures were modified from McConnel et al. ${ }^{5)} 50 \mu \mathrm{g}$ of DNA was digested with a combination of three enzymes; 100 units of RsaI, 50 units of HincI and 50 units of HaeIII. Digested samples were size fractionated onto $1.0 \%$ agarose gel in TAE buffer, 300-800 base pair (bp) fraction was excised, and the DNA purified using the phenol-chloroform procedure. The fragments were ligated into the SmaI site of pUC18. Plasmids were transformed into DH5 $\alpha$ competent cells (Gibco BRL) according to the manufacturer's manual and screened using a (GT) $)_{15}$ oligonucleotide probe which $5^{\prime}$ end-labeled with $\left(\gamma{ }^{32} \mathrm{P}\right)$ ATP. After hybridization overnight at $65^{\circ} \mathrm{C}$ with oligonucleotide and washing twice with $2 \% \mathrm{SDS}+2 \mathrm{xSSC}$ at room temperature and $65^{\circ} \mathrm{C}$, respectively, positive plasmid template was sequenced with a $\mathrm{T} 7$ sequencing kit (Pharmacia) using a 373A DNA Sequencing System (Applied Biosystem Inc.) according to the manufacturer's procedure. Primer pairs were designed by

\footnotetext{
${ }^{*}$ Corresponding Author.
} 
the DNA-Oligo (National Bioscience Inc., Version 4.0) software, complementary to the flanking region of repeat DNA.

\section{Microsatellite Polymorphism}

Microsatellite loci were amplified by the polymerase chain reaction ( $\mathrm{PCR}$ ) to assess the level of collection polymorphism. Polymerase chain reactions were conducted in volumes of $5 \mu \mathrm{l}$, each consisting of $10 \mathrm{ng}$ of template DNA, $2.5 \mu \mathrm{M}$ of the forward primer, $2.5 \mu \mathrm{M}$ of the reverse primer, $1.75 \mu \mathrm{M}$ each of dATP, dCTP, dGTP and dTTP, $1 \times$ reaction buffer $(10 \mathrm{~mm}$ Tris- $\mathrm{HCl}(\mathrm{pH} \quad 8.3), 1 \mathrm{~mm}$ $\mathrm{MgCl}_{2}$, and $50 \mathrm{mM} \mathrm{KCl}$ ), 0.25 units of $T a q$ polymerase (Perkin Elmer), and $0.04 \mu \mathrm{M}$ of reverse or forward primer $5^{\prime}$ end-labeled with $\left[\gamma_{-}{ }^{32} \mathrm{P}\right]$ ATP $(37 \mathrm{kBq} / 10 \mathrm{pmol}$ primer $)$ using T4 polynucleotide kinase (Pharmacia). Amplification cycles consisted of seven cycles of one min at $94^{\circ} \mathrm{C}$, $30 \mathrm{~s}$ at the optimal $T_{a n n}, 30 \mathrm{~s}$ at $72^{\circ} \mathrm{C}$ and followed by $33 \mathrm{cy}$ cles of $30 \mathrm{~s}$ at $90^{\circ} \mathrm{C}, 30 \mathrm{~s}$ at the optimal $T_{a n n}, 30 \mathrm{~s}$ at $72^{\circ} \mathrm{C}$. One part of deionized formamide stop dye was added to each reaction vessel and followed by denaturation at $90^{\circ} \mathrm{C}$ for 15 minutes. About 2-5 $\mu l$ of each sample was loaded onto a $6 \%$ polyacrylamide urea gel, and the alleles were sized relative to a sequence ladder generated from M13mp18. The gel was exposed to X-ray film overnight at $-80^{\circ} \mathrm{C}$.

For each species the number of alleles present, number of effective alleles and observed heterozygosity were calculated. Genotype frequencies in each species at each locus were tested for conformity to Hardy-Weinberg equilibrium. The heterogeneity of allele frequencies was tested using $\chi^{2}$ value, then used to calculated Nei's standard genetic distance using the PHYLIP computer package. ${ }^{6}$ ) Allele frequencies were bootstrapped over 1000 times, and the resultant data set were used to calculate 1000 genetic distance for constructing of UPGMA dendrogram.

\section{Results}

Abundance of $(G T)_{\mathrm{n}}$ and Sequence of Seriola Dumerili Microsatellites

In the present study, a partial genomic library composed of approximately 1500 clones were constructed. A total of 44 microsatellite from those clones were isolated and sequenced, and 24 clones contain (GT) or (CA) repeats. Since the average size of the clonal inserts was about 400 base pairs (bp), the total number of base pairs analyzed were estimated to be $6.0 \times 10^{5} \mathrm{bp}$. Assuming that $(\mathrm{GT})_{n}$ sequences are distributed evenly throughout the $S$. dumerili genome and the haploid genome size was $6 \times 10^{9}$ (reviewed by McConnel et al., ${ }^{5)}$ we estimate that $2.4 \times 10^{5}(\mathrm{GT})_{n}$ loci are represent in the $S$. dumerili genome and the average distance between neighboring microsatellites is $25 \mathrm{kbp}$.

According to Weber, ${ }^{16}$ the cloned microsatellites can be divided into three categories, perfect, imperfect and compound. The present study provided microsatelites with 16 $(\mathrm{GT})_{n}, 6(\mathrm{CA})_{n}$ and two compound repeats. Based on the type of repeats, the microsatellite sequences can be categorized into eleven perfect repeats $(45.83 \%)$, eleven imperfect repeats $(45.83 \%)$, and two compound imperfect repeats $(8.33 \%$ ) (Fig. 1). Sequencing analysis of positive clones revealed one perfect sequence of four nucleotides

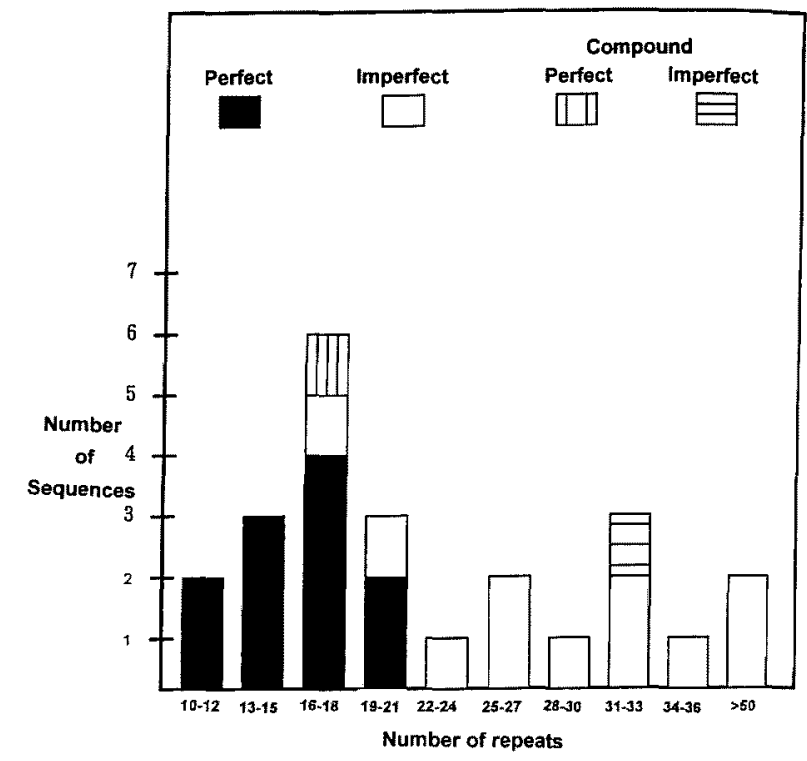

Fig. 1. Frequency of repeat sequences as flanking regions of the greater amberjack microsatellite.

(AGGA) repeats, two uninterrupted series of (GT) repeats, two perfect series of (CA) repeats, three imperfect structures of (GT) repeats with Adenine nucleotide intervening and one imperfect sequence of $(\mathrm{CA})$ repeats. The largest size of (GT) repeats found in this study was categorized in size of $>50$ nucleotide repeats.

\section{Genetic Variability of Four Species of Genus Seriola}

Each microsatellite locus is flanked by a unique sequence. Since that sequence is known, the primers can be synthesized complementary to those flanking sequence regions. These primer sets were used to the Polymerase Chain Reaction (PCR) to amplify a segment that lies between two regions of a known sequence in a series of synthetic reactions that are catalyzed by a DNA polymerase. From this study, nine PCR primers were designed according to DNA sequence flanking repeat region. A preliminary screening of primer revealed six of those primers ( $S d n$ $01^{*}, S d n-03^{*}, S d n-06^{*}, S d n-07^{*}, S d n-08^{*}$ and $\left.S d n-09^{*}\right)$ provided scorable product in $S$. dumerili samples (see Fig. 2). Four microsatellites were polymorphic ( $S d n-06^{*}, S d n$ $07^{*}, S d n-08^{*}$ and $\left.S d n-09^{*}\right)$ and two others were monomorphic $\left(S d n-01^{*}\right.$ and $\left.S d n-03^{*}\right)$ (Table 1), so consequently they were not considered further.

Allelic segregation of four microsatellite loci was also examined in three other species of genus Seriola ( $S$. quinqueradiata, S. lalandi, S. rivoliana), and two species of other genera (Decapterus maruadsi and Uraspis helvola). Three of those four primers were polymorphic for species of the same genus Seriola, however no primer sets are available for amplification DNA from species of other genera. Genotype frequencies observed of all species tested, except in $S$. rivoliana, at all loci were in agreement with expected Mendelian equations. The number of alleles, the number of effective alleles and heterozygosities ranged from 2-16, $1.7-10.8$ and $0.350-0.800$, respectively, across the three loci and four species (Table 2). Clear differences of allele 


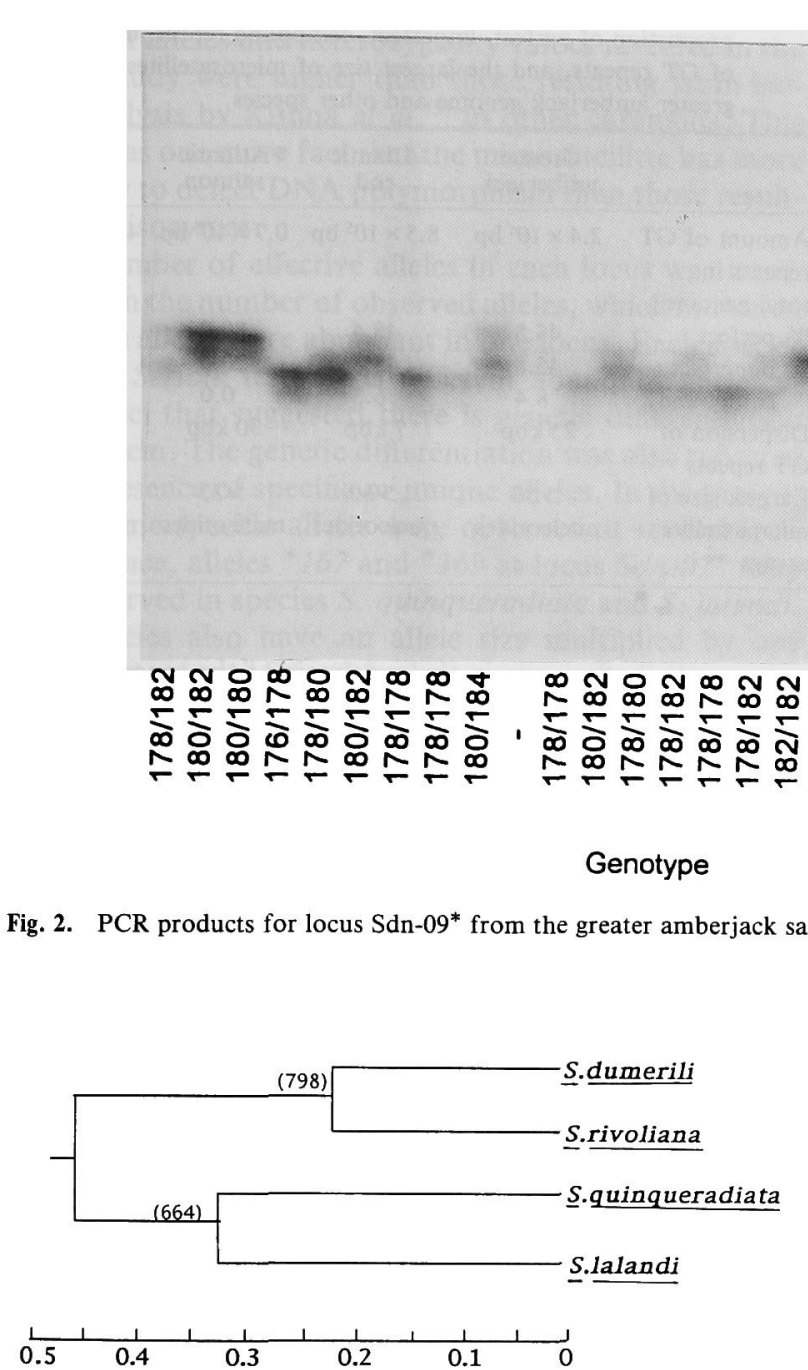

Fig. 3. UPGMA dendrogram of Nei's genetic distance of three loci among four species of genus Seriola.

Values in the brackets are number of times that occurred in 1000 dendrograms generated from 1000 genetic distances by bootstrapping gene frequencies.
A C G T

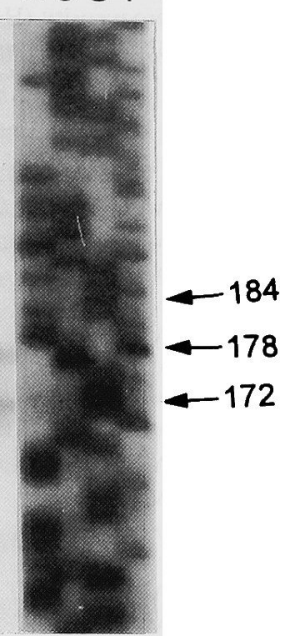

Fig. 2. PCR products for locus Sdn- $09^{*}$ from the greater amberjack samples, with a sequence ladder generated from M13mp18 (at the right side).

frequency distributions were observed between species at three loci. Loci $S d n-07^{*}$ and $S d n-09^{*}$ showed a high level of differentiation between species, while locus $S d n-06^{*}$ revealed low differentiation between species samples. Some species specific alleles were also observed in several loci. Genetic distance calculated based on the three loci show clearly classification among the species samples (Fig. 3).

\section{Discussion}

Abundance of $(G T)_{\mathrm{n}}$ and Sequence of Seriola Dumerili Microsatellites

The feasibility of using library, as matrices assembled into the maps of the genome from which they were originally derived, was established in the late 1970s, as well as proposed by Maniatis et al. ${ }^{7)}$ They have devised a strategy

Table 1. PCR product size ${ }^{+}$, primer sequences ${ }^{+}$and optimal annealing temperature ${ }^{+}$for PCR amplification of the six $S$. dumerili microsatellite loci

\begin{tabular}{|c|c|c|c|}
\hline Locus & Size (bp) & Primer Sequence ('5-'3) & annealing temperature $\left({ }^{\circ} \mathrm{C}\right)$ \\
\hline \multirow[t]{2}{*}{$S d n-01^{*}$} & *242 & F:GGACTGTGAGATGAGGTTGA & 51.7 \\
\hline & & R:GACTTTGGGTTGGCACGAGA & \\
\hline \multirow[t]{2}{*}{$S d n-03^{*}$} & ${ }^{*} 216$ & F:AGTGAGGCAAGCATAGGACAG & 54.5 \\
\hline & & R:AGGTGGGAAAGCATTATCAG & \\
\hline \multirow[t]{2}{*}{$S d n-06^{*}$} & ${ }^{*} 311$ & F:GGGTTTGTGCTGTGAGTG & 52.1 \\
\hline & & R:TCCGTCTGTCTTTTCCTGT & \\
\hline \multirow[t]{2}{*}{$S d n-07^{*}$} & ${ }^{*} 140$ & F:CTTGCTCGTCTCATCACCTC & 48.7 \\
\hline & & R:AACGGCTGGTAAATAGTC & \\
\hline \multirow[t]{2}{*}{$S d n-08^{*}$} & ${ }^{*} 152$ & F:AGTATAGCCCGCATTCCA & 47.0 \\
\hline & & R:TGACTTTCTTCTTTCGTT & \\
\hline \multirow[t]{2}{*}{$S d n-09^{*}$} & ${ }^{*} 181$ & F:GTGTAGCCTATTTGTGTGTT & 50.3 \\
\hline & & R:TTACTTATCTGTCCGCAGCC & \\
\hline
\end{tabular}

+) Result of computer analysis using DNA-Oligo Ver. 4.0 software.

$F=$ forward, $R=$ reverse. 
Table 2. Number of alleles (NA), number of effective alleles (EA) and observed heterozygosity (Ho) in four species of Seriola at three loci $\left(S d n-06^{*}, S d n-07^{*}\right.$, and $\left.S d n-09^{*}\right)$

\begin{tabular}{|c|c|c|c|c|c|}
\hline \multirow{2}{*}{ Species } & & \multicolumn{4}{|c|}{ Microsatellite loci } \\
\hline & & $S d n-06^{*}$ & $S d n-07^{*}$ & $S d n-09^{*}$ & Average \\
\hline S. dumerili & NA & 7 & 12 & 6 & 8.3 \\
\hline \multirow[t]{3}{*}{$(n=40)$} & $\mathrm{EA}$ & 3.8 & 7.5 & 3.4 & 4.9 \\
\hline & Ho & 0.700 & 0.800 & 0.575 & 0.691 \\
\hline & $\mathrm{He}$ & 0.733 & 0.868 & 0.712 & 0.771 \\
\hline \multirow{4}{*}{$\begin{array}{l}\text { S. rivoliana } \\
(n=20)\end{array}$} & NA & 6 & 6 & 5 & 5.7 \\
\hline & $\mathrm{EA}$ & 2.3 & 3.6 & 2.6 & 2.8 \\
\hline & Ho & 0.400 & 0.650 & 0.350 & 0.467 \\
\hline & $\mathrm{He}$ & 0.563 & 0.726 & 0.619 & 0.636 \\
\hline \multirow{4}{*}{$\begin{array}{l}\text { S. quinqueradiata } \\
(n=40)\end{array}$} & NA & 9 & 2 & 13 & 8.0 \\
\hline & $\mathrm{EA}$ & 4.4 & 1.7 & 6.4 & 4.2 \\
\hline & Ho & 0.700 & 0.514 & 0.750 & 0.655 \\
\hline & $\mathrm{He}$ & 0.773 & 0.428 & 0.844 & 0.682 \\
\hline \multirow{4}{*}{$\begin{array}{l}\text { S. lalndi } \\
(n=40)\end{array}$} & NA & 9 & 14 & 16 & 13.0 \\
\hline & EA & 5.2 & 10.8 & 8.5 & 8.2 \\
\hline & Ho & 0.658 & 0.759 & 0.800 & 0.739 \\
\hline & $\mathrm{He}$ & 0.807 & 0.908 & 0.883 & 0.866 \\
\hline
\end{tabular}

to generate and clone large numbers of random fragments of mammalian genomic DNA. Recently, this approach has also been used for other species, including fish. Dinucleotide microsatellite repeat sequences appear to be abundant in the $S$. dumerili genomes. On the basis of our estimate, dinucleotide repeats of $S$. dumerili, present at intervals of $25 \mathrm{kbp}$, are more abundant than in the Atlantic salmon, ${ }^{9)}$ however less abundant than in blue gill, ${ }^{10)}$ brown trout ${ }^{11)}$ and Atlantic $\operatorname{cod}^{12)}$ (Table 3). These differences may be due to the varying stringency of screening used. From the number repeats that resulted, we recorded that there were over 50 bp repeats, whereas similar results have been obtained in Atlantic cod and rainbow trout. ${ }^{12)}$ Nevertheless, according to Brooker et al. ${ }^{12)}$ there is no evidence in the literature if screening at a lower stringency should enhance detection of long, short and more degenerate dinucleotide arrays. Further, the authors suggested that the differences on array length may be due to an effect of temperature on DNA replication.

Utilization of $(\mathrm{GT})_{n}$ repeats in this study was based on the knowledge that $(\mathrm{GT})_{n}$ microsatellites were more predominant than the next abundant type, namely (GA)n or other types in all animal species. ${ }^{13)}$ In this case, we estimated $S$. dumerili has approximately $2.4 \times 10^{5}(\mathrm{GT})_{n}$ repeats, a relatively large number, which is appropriate with Wright's suggestion in eukaryotic genomes. Of those $(\mathrm{GT})_{n}$ repeats, the independent microsatellite arrays isolated were identified as perfect $(45.8 \%)$, imperfect $(45.8 \%)$, and compound imperfect $(8.4 \%)$ repeats. When compared with other fish, the proportion of the perfect and imperfect repeats isolated from $S$. dumerili genome (50:50) most closely resembled the Atlantic cod (52:48) than microsatellites from the rainbow trout (64:36) and Atlantic salmon $(80: 20) .^{12)}$

Further, eight percent of the smallest class of sequences in $S$. dumerili were found, and the most common size class for $S$. dumerili microsatellites would be at least 16-18 dinucleotides in length (25\%), approximately half the second-most common size class of cod. ${ }^{14)}$ According to Sambrook $e{ }^{a l}{ }^{8)}$ this case generally has one of the following
Table 3. Comparison of the amount of microsatellite arrays, proportion of perfect, imperfect, compound repeat, dispersion of GT repeats, and the largest size of microsatellites between greater amberjack genome and other species

\begin{tabular}{lcccc} 
& $\begin{array}{c}\text { Greater } \\
\text { amberjack }\end{array}$ & $\begin{array}{c}\text { Atlantic } \\
\text { cod }\end{array}$ & $\begin{array}{c}\text { Atlantic } \\
\text { salmon }\end{array}$ & $\begin{array}{l}\text { Rainbow } \\
\text { trout }\end{array}$ \\
\hline $\begin{array}{lcccc}\text { Amount of GT } \\
\text { repeats in }\end{array}$ & $2.4 \times 10^{5} \mathrm{bp}$ & $8.5 \times 10^{5} \mathrm{bp}$ & $0.7 \times 10^{5} \mathrm{bp}$ & $4.3 \times 10^{5} \mathrm{bp}$ \\
total genome & & & & \\
$\%$ perfect & 45.8 & 48.4 & 80.0 & 56.9 \\
$\%$ imperfect & 45.8 & 45.3 & 20.0 & 31.4 \\
$\%$ compound & 8.4 & 6.3 & 0.0 & 11.7 \\
$\begin{array}{l}\text { Dispersion of } \\
\text { GT repeats }\end{array}$ & $25 \mathrm{kbp}$ & $7 \mathrm{kbp}$ & $90 \mathrm{kbp}$ & $14 \mathrm{kbp}$ \\
Largest size of & $>50$ & $>60$ & $>33$ & $>60$ \\
microsatellite & nucleotides & nucleotides & nucleotides & nucleotides \\
arrays & & & &
\end{tabular}

causes: (1) inefficiency in the synthesis of the second strand of DNA, resulting in double-stranded molecules that contain gaps or traces of mRNA, or (2) small pieces of DNA (fragments of DNA or undigested oligomers of linkers) contaminate the final preparation of DNA. However, we suggested that this is more likely due to our classifying microsatellites shorter than ten repeats as part of a larger degenerate microsatellite array, and the criteria which define an imperfect repeat are alliterated to incorporate three intervening bases as Weber's definition. ${ }^{16)}$ We did not calculate those repeats as microsatellite sequences, while Brooker et $a l .^{12)}$ used 4-5 intervening bases adjacent. The absolute lengths of the largest microsatellite arrays in $S$. dumerili ( $>50$ repeats) seems to be similar to those reported in $\operatorname{cod}$ ( $>60$ repeats), rainbow trout ( $>60$ repeats) and longer than Atlantic salmon ( $>33$ repeats) (Table 3 ). The length and class of microsatellites become important when considering the level of polymorphim that one can expect for a given locus. In humans, it has been shown that the level of polymorphism is proportional to the length of uninterrupted repeats. ${ }^{14)}$ Therefore, it is more informative to obtain the largest of number uninterrupted repeats possible.

\section{Genetic Variability of Four Species of Genus Seriola}

Three of the four microsatellite loci have polymorphism with variability in allele size for other species of genus Seriola. The allele size was multipled by two nucleotides, except in species $S$. quinqueradiata and $S$. rivoliana for locus $S d n-09^{*}$. The particularly exciting finding from this study is the ability of the $S$. dumerili primers, $S d n-06^{*}$, $S d n-07^{*}$ and $S d n-09^{*}$, to amplify presumably homologous microsatellite loci in three other carangid species of genus Seriola, even not for carangid species from other genera. This became important due to the high initial cost of developing markers for a particular species. Similar results have also been obtained in salmonid species, ${ }^{5}$ and with application for the Atlantic cod primers to other gadiid. ${ }^{12)}$

Generally, species $S$. lalandi has relatively more variability in the number of alleles and heterozygosity value than the other species tested. The average number of alleles and observed heterozygosity values of species $S$. lalandi were 13.0 alleles and 0.739 , then followed by species $S$. dumerili (8.3 alleles; $\mathrm{Ho}=0.692$ ), S. quinqueradiata (8.0 alleles; 
$\mathrm{Ho}=0.650)$ and $S$. rivoliana $(5.7$ alleles; $\mathrm{Ho}=0.467)$. The number of alleles and heterozygosity values revealed in the present study were higher than those resulting from isozyme analysis by Kijima et $a l^{15)}$ in other carangids. This feature was one more fact that the microsatellitte has more sensitivity to detect DNA polymorphism than those resulting from isozymes.

The number of effective alleles in each locus was much lower than the number of observed alleles, which indicated that some alleles were abundant in one locus. Each species of genus Seriola tested has different abundant alleles at several loci that suggested there is genetic differentiation among them. The genetic differentiation was also reflected by the presence of specific or unique alleles. In the present study, some specific alleles were observed at several loci. For instance, alleles ${ }^{*} 167$ and ${ }^{*} 169$ at locus $S d n-07^{*}$ were only observed in species $S$. quinqueradiata and $S$. lalandi. Both species also have an allele size multiplied by one nucleotide at locus $S d n-09^{*}$ whereas those alleles were not observed in species $S$. dumerili and $S$. rivoliana. Further, the similar patterns of allele distribution between $S$. dumerili with $S$. rivoliana, and $S$. quinqueradiata with $S$. lalandi were observed in this study. The similarity between those species was also found morphologically. According to Gushiken, ") the body form of $S$. quinqueradiata was similar to $S$. lalandi, while species $S$. dumerili has the same body form as $S$. rivoliana. Visually, the differentiation between $S$. quinqueradiata and $S$. lalandi was in the shape of the maxilla, and the differentiation between $S$. dumerili with $S$. rivoliana was in the length of the anterior of the second dorsal fin. It seems likely that their genetic divergence was reflected in the morphological ones. However, this feature should be observed further using more loci and a larger number of samples.

The observed heterozygosity varied at all loci, ranging between $0.400-0.700,0.514-0.800,0.350-0.800$ at loci $S d n-06^{*}, S d n-07^{*}$ and $S d n-09^{*}$, respectively. Generally, species $S$. rivoliana has a lower value of observed heterozygosities than others. The relatively low heterozygosity values revealed in species $S$. rivoliana may be due to the limited number of samples used. Even though, according to the value of $\mathrm{Ho} / \mathrm{He}$, the lowest value was in species $S$. rivoliana $(0.566)$ which indicated there is a possibility of reducing the genetic variability or the presence of null allele which was not detectable due mutation in the sequence of the primer set. Genetic change of their population structure in the natural population, may also influence this phenomena, and therefore this should be considered in the future. In addition, we suggested that differentiation of their variability may also have correlation with their distribution or population size. Notably, $S$. rivoliana is more rarely found than other species of genus Seriola in Japan, which also indicates that this species has a smaller population size than the others. Since the microsatellite mutation rate is estimated to be $10^{-4},{ }^{17}$ the effective population sizes revealed in the present study are about 3,$150 ; 9,251$; 11,841 and 17,099 fish, respectively for $S$. rivoliana, $S$. quinqueradiata, $S$. dumerili and $S$. lalandi. Therefore, the probability of inbreeding in $S$. rivoliana is higher than in the three other species due to their rarely meeting with other populations.

Further, the lowest value of genetic distance was ob- served between species $S$. dumerili and $S$. rivoliana $(0.442)$, then followed by distance between species $S$. quinqueradiat $a$ and $S$. lalandi $(0.643)$. About $79.80 \%$ and $66.40 \%$ of the similarity were observed between species $S$. dumerili-S. rivoliana and $S$. quinqueradiata-S. lalandi (see Fig. 3). This result agreed with the taxonomic arrangement as described by Gushiken ${ }^{1)}$ where species $S$. dumerili was closely related to species $S$. rivoliana, while species $S$. quinqueradiata was similar to species $S$. lalandi. Even though, this similarity was not significant, the genetic differentiation between those species pairs was still observed in the present study. This phenomenon showed that microsatellite can also be used as markers for phylogenetic relationship, as well as other applications in population studies.

\section{References}

1) S. Gushiken: Revision of the carangid fishes of Japan. Galaxea, 2, 135-264 (1983).

2) Food and Agriculture Organization of The United Nations: Expert consultation on utilization and conservation of aquatic genetic resources. Grottaferrata, Italia, 9-13 November 1992, pp. 1-18.

3) L. K. Park and P. Moran: Developments in molecular genetic techniques in fisheries, in "Molecular Genetics in Fisheries" (ed. by G. R. Carvalho and T. J. Pitcher), Chapmann \& Hall, London, 1995, pp. 1-28.

4) A. S. Haris, S. Bieger, R. W. Doyle, and J. M. Wright: DNA fingerprinting of tilapia, Oreochromis niloticus, and its application to aquaculture genetics. Aquaculture, 92, 157-163 (1992).

5) S. McConnel, L.Hamilton, D. Moris, D. Cook, D. Paquet, P. Bentzen, and J. Wright: Isolation of salmonid microsatellite loci and their application to the population genetics of Canadian east coast stocks of Atlantic salmon. Aquaculture, 137, 19-30 (1995).

6) J. Felsenstein: PHYLIP (PHYLogeny Interference Package), versions 3.5c. University of Wahington, Seattle, 1993.

7) T. Maniatis, R.C. Hardison, E. Lacy, J. Lauer, C. O'Connel, D. Quon, G. K. Sim, and A. Efstratiadis.: The isolation of structural genes frm libraries eucaryotic DNA. Cell, 15, 687 (1978).

8) J. Sambrook, E. F. Fritsch, and T. Maniatis: Molecular Cloning: A Laborataory Manual, 2nd, ed. Cold spring Harbor Laboratory Press, New York, 1989, p.I.47.

9) A. I. Slettan, I. Olsaker, and O. Lic: Isolation and characterization of $(\mathrm{GT})_{n}$ repetitive sequences from Atlantic salmon, Salmo salar L. Animal Genetics, 24, 195-197 (1993).

10) J. K. Colbourne, B. D. Neff., J. M. Wright, and M. R. Gross: DNA fingerprinting of bluegill sunfish (Lepomis macrochirus) using (GT) ${ }_{n}$ microsatellites and its potential for assessment of mating success. Canad. J. Fish Aquatic Sci., 53, 342-349 (1996).

11) A. Estoup, P. Presa, F. Krieg, D. Vamman, and R. Guyomard: $(\mathrm{CT})_{n}$ and $(\mathrm{GT})_{n}$ microsatellites; A new class of genetic markers for Salmo trutta L. (brown trout). Heredity, 71, 488-496 (1993).

12) A. L. Brooker, D. Cook, P. Bentzen, J. M. Wright, and R. W. Doyle: Organization of microsatelites differs between mammals and coldwater teleost fish. Canad. J. Fish Aquatic Sci., 51, 1959-1965 (1994).

13) S. Brenner, G. Elgar, R. Sandford, A. Macrae, H. Venkatesh, and S. Aparico: Characterization of puffr fish ( $F u g u$ ) as a compact model vertebrate genome. Nature, 366, 265-268 (1993).

14) C. Rico, K. M. Ibrahim, I. Rico, and G. M. Hewitht.. Stock composition in North Atlantic populations of whiting using microsatellite markers. J. Fish Biol., 51, 462-475 (1997).

15) A. Kijima, N. Taniguchi, and A. Ochiai: Genetic divergence and relationship among fifteen species of genera Trachurus, Selar and Selaroides. Jap. J. Ichthyol., 35, 2, 167-175 (1988).

16) J. L. Weber: Informativeness of human $(\mathrm{dC}-\mathrm{dA})_{n}(\mathrm{dG}-\mathrm{dT})_{n}$ polymorphisms. Genomics, 7, 524-530 (1990).

17) F. J. G. De leon, L. Chikhi, and F. Bonhomne: Microsatellite polymorphism and population subdivision in natural populations of European sea bass, Dicentrarchus labrax (Linnaeus, 1758). Molecular Ecologv. 6. 51-62 (1997). 